\title{
VLBI for Gravity Probe B. VI., the Orbit of IM Pegasi and the Location of the Source of Radio Emission
}

\section{Citation}

Ransom, R. R., N. Bartel, M. F. Bietenholz, D. E. Lebach, J.-F. Lestrade, M. I. Ratner, and I. I. Shapiro. 2012. "VLBI for Gravity Probe B. VI., the Orbit of IM Pegasi and the Location of the Source of Radio Emission." The Astrophysical Journal Supplement Series 201, no. 1: 1-9.

\section{Published Version}

doi:10.1088/0067-0049/201/1/6

\section{Permanent link}

http://nrs.harvard.edu/urn-3:HUL.InstRepos:11859329

\section{Terms of Use}

This article was downloaded from Harvard University's DASH repository, and is made available under the terms and conditions applicable to Other Posted Material, as set forth at http:// nrs.harvard.edu/urn-3:HUL.InstRepos:dash.current.terms-of-use\#LAA

\section{Share Your Story}

The Harvard community has made this article openly available.

Please share how this access benefits you. Submit a story.

Accessibility 


\title{
VLBI FOR GRAVITY PROBE B. VI. THE ORBIT OF IM PEGASI AND THE LOCATION OF THE SOURCE OF RADIO EMISSION
}

\author{
R. R. Ransom ${ }^{1,4}$, N. Bartel ${ }^{1}$, M. F. Bietenholz ${ }^{1,5}$, D. E. LebaCh ${ }^{2}$, J.-F. Lestrade ${ }^{3}$, M. I. RATneR ${ }^{2}$, And I. I. Shapiro ${ }^{2}$ \\ ${ }^{1}$ Department of Physics and Astronomy, York University, 4700 Keele Street, Toronto, ON, M3J 1P3, Canada \\ ${ }^{2}$ Harvard-Smithsonian Center for Astrophysics, 60 Garden Street, Cambridge, MA 02138, USA \\ ${ }^{3}$ Observatoire de Paris/LERMA, 77 Av. Denfert Rochereau, F-75014 Paris, France \\ Received 2011 May 31; accepted 2012 January 18; published 2012 June 7
}

\begin{abstract}
We present a physical interpretation for the locations of the sources of radio emission in IM Pegasi (IM Peg, HR 8703), the guide star for the NASA/Stanford relativity mission Gravity Probe B. This emission is seen in each of our 35 epochs of $8.4 \mathrm{GHz}$ very long baseline interferometry observations taken from 1997 to 2005 . We found that the mean position of the radio emission is at or near the projected center of the primary to within about $27 \%$ of its radius, identifying this active star as the radio emitter. The positions of the radio brightness peaks are scattered across the disk of the primary and slightly beyond, preferentially along an axis with position angle, P.A. $=-38^{\circ} \pm 8^{\circ}$, which is closely aligned with the sky projections of the orbit normal (P.A. $=-49.5 \pm 8.6$ ) and the expected spin axis of the primary. Comparison with simulations suggests that brightness peaks are $3.6_{-0.7}^{+0.4}$ times more likely to occur (per unit surface area) near the pole regions of the primary (latitude, $|\lambda| \geqslant 70^{\circ}$ ) than near the equator $\left(|\lambda| \leqslant 20^{\circ}\right)$, and to also occur close to the surface with $\sim 2 / 3$ of them at altitudes not higher than $25 \%$ of the radius of the primary.
\end{abstract}

Key words: binaries: close - radio continuum: stars - stars: activity - stars: imaging - stars: individual (IM Pegasi) - techniques: interferometric

Online-only material: color figures

\section{INTRODUCTION}

IM Pegasi (IM Peg; HR 8703; HD 216489; FK5 3829) is the radio-bright binary star which served as the guide star for the Gravity Probe B (GP-B) mission, the space-borne relativity experiment developed by NASA and Stanford University to test two predictions of general relativity. This paper is the sixth in a series of seven describing the program of very long baseline interferometry (VLBI) undertaken in support of $G P-B$. In the first paper in the series, we give an introduction to $G P-B$ and to this series (Paper I, Shapiro et al. 2012). In the second and third papers, we report on the structure and its changes of each of the three extragalactic reference sources, 3C 454.3, B2250+194, and B2252+172 (Paper II, Ransom et al. 2012), and on the degree of astrometric stability of the "core" of 3C 454.3 in two extragalactic celestial reference frames (Paper III, Bartel et al. 2012). In the fourth and fifth papers, we describe our astrometric analysis technique (Paper IV, Lebach et al. 2012) and present our result on the proper motion, parallax, and orbit of IM Peg (Paper V, Ratner et al. 2012). In this paper (Paper VI), we discuss the locations of the sources of radio emission in the IM Peg system and give a physical interpretation of the sources' origins. In the last paper of our series (Paper VII, Bietenholz et al. 2012), we discuss the radio images of IM Peg and include a movie of this star's changes over the duration of our 8.5 year observing program.

IM Peg is a close binary with an orbital period of $\sim 24.65$ days and an essentially circular orbit with an eccentricity of $0.006 \pm 0.007$ (Berdyugina et al. 1999). It is classified by Hall

\footnotetext{
4 Now at Okanagan College, 583 Duncan Avenue West, Penticton, BC, V2A 2K8, Canada, and also at the National Research Council of Canada, Herzberg Institute of Astrophysics, Dominion Radio Astrophysical Observatory, PO Box 248, Penticton, BC, V2A 6K3, Canada.

5 Now also at Hartebeesthoek Radio Astronomy Observatory, PO Box 443, Krugersdorp 1740, South Africa.
}

(1976) as an RS CVn. The system is at a distance of $96.4 \pm 0.7$ pc (Paper V; see also ESA 1997) and has an inclination of $65^{\circ} \leqslant i \leqslant 80^{\circ}$ (Berdyugina et al. 1999; Lebach et al. 1999). The primary is a K2 III star (Berdyugina et al. 1999) which is magnetically active, showing bright emission features (e.g., Ca II $\mathrm{H}$ and $\mathrm{K}, \mathrm{Mg}$ II $\mathrm{H}$ and $\mathrm{K}, \mathrm{C}$ IV) that are presumably produced by high-temperature species in its chromosphere and transition region (Huenemoerder et al. 1990; Dempsey et al. 1996; Olah et al. 1998). In addition, Doppler optical images of the photosphere of the primary exhibit large, relatively dim "spot" regions, covering collectively $>15 \%$ of the visible stellar surface (Berdyugina et al. 2000). The sunlike secondary is $\sim 60$ times less luminous in the optical than the primary, and has also been detected spectroscopically (Marsden et al. 2005).

Radio emission from IM Peg was first detected by Spangler et al. (1977). Since then, IM Peg has been included in two radio surveys of RS CVn systems (Morris \& Mutel 1988; Drake et al. 1989), but few details of its radio properties are published. Its flux density at centimeter wavelengths has ranged between $\sim 0.2 \mathrm{mJy}$ and $\sim 80 \mathrm{mJy}$ (Paper I; Lebach et al. 1999; Boboltz et al. 2003), and can be highly variable on subhour timescales (Lebach et al. 1999). VLBI observations of IM Peg were made in the early 1990s as part of an astrometric program to link the Hipparcos optical reference frame to the extragalactic radio reference frame (Lestrade et al. 1995, 1999), but no image of IM Peg from this program was published.

Models for the microwave radio emission of RS CVn binaries suggest three possible source regions for the emission within the system: (1) magnetic-loop structures attached to one stellar component, namely, the active subgiant or giant, in the binary (e.g., Mutel et al. 1985; Franciosini et al. 1999); (2) a joint magnetosphere for the two components of the binary (e.g., Uchida \& Sakurai 1983; Ransom et al. 2002); and (3) the region between the two components (Lestrade 1996). Multi-epoch astrometric VLBI observations can potentially distinguish among 
Table 1

Properties and Previously Determined Orbital Parameters of IM Peg

\begin{tabular}{|c|c|c|c|}
\hline Parameter & \multicolumn{2}{|c|}{ Value } & Reference $^{\mathrm{a}}$ \\
\hline Trigonometric parallax (mas) & \multicolumn{2}{|c|}{$10.33 \pm 0.76,10.370 \pm 0.074$} & 1,2 \\
\hline Distance $(\mathrm{pc})$ & \multicolumn{2}{|c|}{$96.8_{-6.2}^{+7.7}, 96.4 \pm 0.7$} & 1,2 \\
\hline \multicolumn{4}{|c|}{ Stellar properties $^{b}$} \\
\hline Mass $\left(M_{\odot}\right)$ & $1.8 \pm 0.2$ & $1.0 \pm 0.1$ & 3,3 \\
\hline Spectral type & K2 III & G V ? ${ }^{\mathrm{c}}$ & 4,3 \\
\hline$T_{\text {eff }}(\mathrm{K})$ & $4550 \pm 50$ & $5650 \pm 200^{c}$ & 4,3 \\
\hline Radius $\left(R_{\odot}\right)$ & $13.3 \pm 0.6$ & $1.00 \pm 0.07^{\mathrm{c}}$ & 4,3 \\
\hline Radius (mas) ${ }^{\mathrm{d}}$ & $0.64 \pm 0.03$ & $0.048 \pm 0.004^{\mathrm{c}}$ & 4,3 \\
\hline \multicolumn{4}{|c|}{ Orbital elements $^{b}$} \\
\hline$a \sin i\left(R_{\odot}\right)$ & $16.70 \pm 0.02$ & $30.34 \pm 0.03$ & 3,3 \\
\hline$a \sin i(\mathrm{mas})^{\mathrm{d}}$ & 0.806 & 1.464 & \\
\hline$P$ (days) & \multicolumn{2}{|c|}{$24.64877 \pm 0.00003$} & 3 \\
\hline$i\left(^{\circ}\right)$ & \multicolumn{2}{|c|}{$65 \ldots 80,>55$} & 4,5 \\
\hline$e$ & \multicolumn{2}{|c|}{0.0 (assumed) } & 4 \\
\hline$T_{\text {conj }}(\mathrm{HJD})^{\mathrm{e}}$ & \multicolumn{2}{|c|}{$2450342.905 \pm 0.004$} & 3 \\
\hline
\end{tabular}

Notes.

a The first reference is for the first entry, second reference, if present, is for the second entry.

b Two entries for lines 3-9 correspond to the two stars of the binary system, with entries for the primary listed first.

c The spectral type, effective temperature, and radius of the secondary are inferred from the flux ratios (at two wavelengths) of the two stellar components and the values for the radius and effective temperature of the primary under the assumption that the secondary is a main-sequence star.

d Computed for a system distance of $96.4 \pm 0.7 \mathrm{pc}$. The uncertainty in the $a \sin i$ value in $R_{\odot}$ units is not propagated into mas, since the uncertainty in the inclination is the dominant source of error in any spectroscopic determination of the semimajor axis.

${ }^{\mathrm{e}}$ Heliocentric time of conjunction with the K2 III primary behind the secondary. References. (1) Hipparcos Catalogue (ESA 1997); (2) VLBI (Paper V); (3) Marsden et al. 2005; (4) Berdyugina et al. 1999, $(e=0.006 \pm 0.007)$; (5) Lebach et al. 1999.

these scenarios. In the related case of the close binary in the Algol system, Lestrade et al. (1993) were able, with astrometric VLBI from four epochs, to identify the cooler $\mathrm{K}$ subgiant star, and not its B dwarf companion or the intermediary region, as the likely source of the radio emission. Until now, no such identification has been made for any RS CVn system.

A total of 35 additional sessions of astrometric VLBI observations of IM Peg were conducted between 1997 and 2005 in support of $G P-B$. Consequently, IM Peg is now more extensively observed at centimeter wavelengths than any other binary. In Section 2 below, we give the previously determined orbital parameters of IM Peg, and set the stage for a discussion about the source region of the radio emission in the binary system. In Section 3, we give an overview of the VLBI observations and astrometric analysis procedures. In Section 4, we summarize the astrometric results for IM Peg presented in Paper V, focusing in particular on the apparent orbit of the radio emission region and the distribution of the residuals on the sky. We discuss our results in Section 5 and give our conclusions in Section 6.

\section{PREVIOUSLY DETERMINED ORBITAL PARAMETERS OF IM PEG}

Optical spectroscopic and photometric observations provide accurate values for most of the basic physical properties and orbital elements of the IM Peg binary system. Table 1 summarizes these results. The orbits of the primary and secondary stars projected on the sky are particularly relevant to the problem of determining the location of the radio emission within the binary system. If the emission source is closely tied to either of the two stars, then it likely travels nearly the same projected orbital path as that star. However, if the emission source arises primarily in the interbinary region, the source could remain more nearly stationary near the center of mass of the binary.

The rotation of at least the K2 III primary is synchronized with the star's orbit (Olah et al. 1998), which is expected to be circular based on tidal theory (Zahn 1977), and indeed determined to be very nearly circular (Olah et al. 1998; Berdyugina et al. 1999; Marsden et al. 2005). Projecting a circular orbit with system inclination $i \gtrsim 55^{\circ}$ (see Table 1 ) on the sky yields a highly eccentric, elliptically shaped orbit. The semimajor axis lengths of the projected (elliptical) orbits of the primary and secondary, $a_{1}$ and $a_{2}$, respectively, are equal to those of the true (circular) orbits, which are constrained by spectroscopic data. Using the values from such data for $a_{1} \sin i$ and $a_{2} \sin i$ in Table 1 , the narrow range of allowed inclination values $65^{\circ} \leqslant i \leqslant 80^{\circ}$ (Berdyugina et al. 1999), and a system distance of $96.4 \pm 0.7$ pc (Paper V), we deduce that the semimajor axes of the orbits for the primary and secondary in angular units are $a_{1}=0.85 \pm 0.04$ mas and $a_{2}=1.55 \pm 0.06$ mas. These values imply that the maximum dimension of the projected orbit of each star is large enough to be detected with astrometric VLBI and that the projected orbits of the primary and secondary stars are clearly distinguishable. If the radio emission from IM Peg is indeed spatially associated largely or entirely with one of the two stars of the binary, then a projected orbit derived from our astrometric observations can distinguish between these two possibilities.

\section{OVERVIEW OF OBSERVATIONS AND ASTROMETRIC ANALYSIS PROCEDURES}

\subsection{VLBI Observations}

Our 35 sessions of $8.4 \mathrm{GHz}(\lambda=3.6 \mathrm{~cm})$ VLBI observations each used a global VLBI array of between 12 and 16 telescopes, including the full Very Long Baseline Array (VLBA) and the 70 m NASA-JPL Deep Space Network (DSN) telescopes. For each session, we interleaved observations of IM Peg with either two or three extragalactic reference sources nearby on the sky, so that we could employ the phase-referencing technique (e.g., Shapiro et al. 1979; Bartel et al. 1986; Lestrade et al. 1990; Beasley \& Conway 1995) and determine an accurate astrometric position for IM Peg. For a full description of our array, typical observing schedule, and data recording parameters, see Paper II. Other aspects of our observing strategy, e.g., seasonal and orbitphase distributions of our observation sessions, are discussed in Paper V and Paper VII. Since the locations of the sources of radio emission in IM Peg are of special interest in this paper, we emphasize here that we took care in our scheduling to achieve an approximately even distribution of orbital phase, without strong correlations between phase and either year or season.

\subsection{The Astrometric Technique}

The phase-referencing process by which we estimated the position of IM Peg for each observing session included both phase-delay fitting with a Kalman-filter estimator and phasereference mapping (see Paper IV for details). This process improves our astrometric accuracy by allowing us to effectively model the contributions of the troposphere, ionosphere, and the extended structure of IM Peg's radio emission regions 
Table 2

Orbit Parameters of IM Peg Radio Source

\begin{tabular}{lcc}
\hline \hline Parameter & Estimate & Standard Error $^{\mathrm{a}}$ \\
\hline \multicolumn{3}{c}{ The parameters of the linear orbit model } \\
\hline$A_{s \alpha}$ (mas) & -0.59 & 0.10 \\
$A_{s \delta}$ (mas) & -0.66 & 0.11 \\
$A_{c \alpha}$ (mas) & 0.15 & 0.09 \\
$A_{c \delta}$ (mas) & -0.23 & 0.11 \\
\hline \multicolumn{2}{c}{ The equivalent values of the more commonly used orbit parameters } \\
\hline$a$ (mas) $_{\text {Axial ratio }}{ }^{\mathrm{b}}$ & 0.89 & 0.09 \\
$\Omega\left({ }^{\circ}\right)^{\mathrm{c}}$ & 0.30 & 0.13 \\
$T_{\text {conj }}$ (JD) & 40.5 & 8.6 \\
\hline
\end{tabular}

Notes.

a See Paper V for an explanation of our errors.

b The axial ratio, i.e., the ratio of the minor to the major axis, is equal to the absolute value of the cosine of the inclination for our zero-eccentricity orbit. The axial ratio therefore corresponds to an inclination of $73^{\circ} \pm 8^{\circ}$.

${ }^{c}$ Position angle (east of north) of the ascending node. We follow the convention of identifying the ascending node as the one at which the source is receding from us as it passes through the plane of the sky.

(see Section 3.3), in spite of the often low flux density (as low as $\sim 0.2 \mathrm{mJy}$ ) of the stellar radio emission. The final image of IM Peg produced for each session is referenced to the quasistationary component $\mathrm{C} 1$, the $8.4 \mathrm{GHz}$ "core," of 3C 454.3. The identification and stationarity of this reference point are discussed in Papers II and III, respectively.

\subsection{Choosing a Position for IM Peg at Each Epoch}

As mentioned in Paper V, our phase-referenced images reveal three general categories for the radio source structure of IM Peg: (1) single-peaked with the peak located near the center of a marginally extended source, (2) single-peaked with the peak located off center from an elongated source, and (3) double-peaked (or in one case apparently triple-peaked) with a maximum separation between peaks of $\sim 1.5$ mas. An example from each of these categories is given in Paper V.

For the definition of the "observed" position for IM Peg, we considered three possible choices: (1) the position of the brightness peak, interpolated between pixels, of the image for each epoch; (2) the central position of the single elliptical Gaussian fit to the image for each epoch; and (3) the central position of the single fit Gaussian for each single-peaked epoch and the position of the unweighted midpoint between the interpolated brightness peaks for each multiple-peaked epoch.

We then fit the astrometric model described in Section 3.4 below to each set of positions. We obtained the best fit for choice (3): the chi-square per degree of freedom for the resulting weighted least-squares fit was $30 \%$ lower than for the worst fit, that for choice (1). We therefore adopted set (3) as our standard set of 35 IM Peg positions for the estimate of position at epoch, proper motion, parallax, and orbital parameters of IM Peg. Set (3) represents in effect smoothed data, taking into account the extended structure of the source. For the study of the locations of the sources of radio emission in this paper, however, the distribution of the locations of emission peaks is more important, and therefore set (1) is used for such an investigation, as described below in Section 4.3.

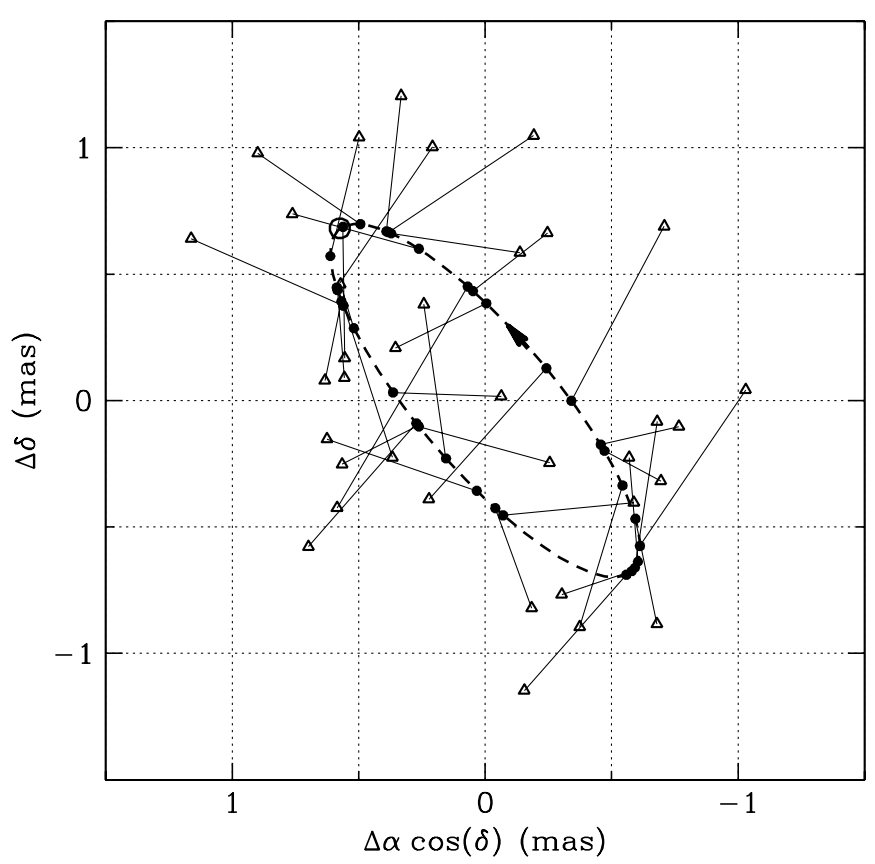

Figure 1. Orbit (dashed ellipse) derived from the nine-parameter fit to the set of 35 positions of the IM Peg radio source. The inferred direction of motion on the sky is counterclockwise, as indicated by the arrow on the ellipse. The ascending node is given as an open circle on the orbit in the northeast. In other words, the part of the ellipse with the arrow on it is closest to Earth. The observed position for each epoch is plotted (with an open triangle) after subtraction of the estimated position at reference epoch, proper motion, and parallax. This position corresponds to the peak of a single Gaussian component fit to the source region or the midpoint between two (or three) local maxima in the source region. A solid line connects each observed position with the corresponding position indicated by a dot on the estimated orbit (see the text).

\subsection{The Astrometric Model}

We fit to the 35 positions of set (3) a nine-parameter model (see also Paper V) describing the motion of the IM Peg radio emission on the sky. The model parameters are the five scalar parameters needed to specify IM Peg's position at a reference epoch, its proper motion, and its parallax, plus four more to specify the projection on the sky of its (assumed) zeroeccentricity orbit of known period. To maintain the linearity of the model in all unknown parameters, we let the orbit parameters for each coordinate $(\alpha$ and $\delta)$ be the amplitudes of the sine $\left(A_{s \alpha}\right.$, $\left.A_{s \delta}\right)$ and cosine $\left(A_{c \alpha}, A_{c \delta}\right)$ terms in orbital phase.

\section{RESULTS}

\subsection{Astrometric Solution}

The full astrometric solution is presented in Table 3 of Paper V. In this paper, we focus on the orbit. For the convenience of the reader and for easier comparison with previously determined orbital parameters, we reproduce in Table 2 the values for the four orbit parameters determined in Paper V. The estimated orbit and the 35 residual position determinations with their corresponding orbit-model-predicted positions are plotted in Figure 1. The inferred direction of motion is counterclockwise as indicated by the arrow. The size, shape, orientation, and "timing" of the orbit do not change significantly if we choose instead set (1) or set (2) of astrometric positions described in Section 3.3. The orbit is also robust against reasonable changes in either the set of epochs included in the fit or the addition to the astrometric model of a constant proper acceleration. A 


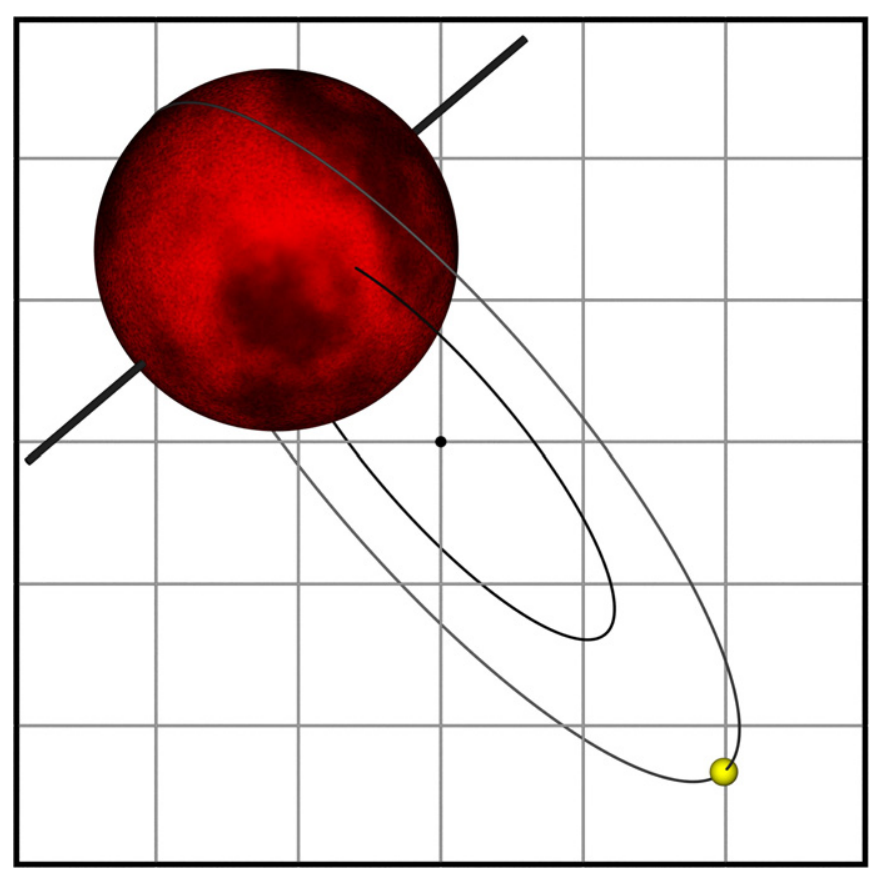

Figure 2. Artist's three-dimensional rendering of the IM Peg binary system as seen from Earth. Grid lines are drawn every 0.5 mas. The primary is the larger red star with dark spots, while the secondary is the smaller yellow star. The projected orbit of the primary is the same as the inferred radio source orbit shown in Figure 1. The size of the orbit of the secondary is computed from that of the radio orbit and the ratio of the component masses given in Table 1. The diameters of the primary and secondary stars correspond to the nominal values given in Table 1. The black dot at the center of the figure represents the center of mass of the binary system. The system is shown with the primary at its ascending node. The spin axis of the primary is indicated by the rod passing through the star's center. It is oriented parallel to the orbit normal (see Section 5). Note that the visible pole of the primary is near the southeast side of the stellar disk.

(A color version of this figure is available in the online journal.)

full discussion of our error analysis is given in Paper V. The orbit of the secondary is obtained from the ratio of the component masses given in Table 1. In Figure 2, we show an artist's three-dimensional rendition of IM Peg with the primary and secondary in their estimated orbits as seen from Earth.

We emphasize three points from Table 2, each of importance for our study of the location of the sources of radio emission.

1. Our estimate of the length of the semimajor axis of $0.89 \pm 0.09$ mas (Paper V) is both statistically significant and consistent within $1 \sigma$ with that of the semimajor axis inferred for the IM Peg primary from optical spectroscopy (see Section 2, Table 1).

2. The ratio of the length of the minor axis to that of the major axis of the projected orbit of $0.30 \pm 0.13$ is relatively small, allowing for a relatively accurate estimate of the position angle (P.A.) of the ascending node, $\Omega=40.5 \pm 8.6$, and hence the P.A. of the projected orbit normal, $\Omega-90^{\circ}=$ $-49.5 \pm 8.6$.

3. The time of conjunction, $T_{\text {conj }}=2450342.56 \pm 0.44 \mathrm{JD}$, implied by our orbit is also consistent within $1 \sigma$ with that derived for the primary from optical spectroscopy (see Table 1).

\subsection{The Mean Location of the Source of the Radio Emission}

In Paper V, we show that the residuals of our nine-parameter astrometric fit to set (3) of the 35 positions of IM Peg scatter about a well-determined orbit on the sky. How does this fit determine the mean location of the radio emission source with respect to the optical primary and secondary of the binary system? With a near zero-eccentricity orbit, the axial ratio of the projected radio orbit corresponds to an inclination of $73^{\circ} \pm 8^{\circ}$. Combining our value for the inclination with the $a \sin i$ estimate of Marsden et al. (2005) for each stellar component (see Table 1) leads to semimajor axes of $0.84 \pm 0.03$ mas for the primary and $1.53 \pm 0.06$ mas for the secondary. Thus, our estimated semimajor axis of the radio orbit of $0.89 \pm 0.09$ mas agrees well with that of the spectroscopic orbit of the primary, but differs significantly from that of the secondary. Furthermore, the time of conjunction, $T_{\text {conj }}$, obtained for the radio orbit is only $0.3 \pm 0.4$ days earlier than that found by Marsden et al. (2005; see Table 1). The estimated radio orbit is thus in the same phase within the error as the spectroscopic orbit. The above difference in $T_{\text {conj }}$ corresponds to a physical offset between the center of the primary and the mean position of the radio emission from our model orbit which is only $0.12 \pm 0.15$ times the radius of the primary and is not significantly different from zero. ${ }^{6}$ That is, the offset is with $1 \sigma$ accuracy likely to be distant from the center by less than $27 \%$ of the radius of the primary. We therefore have strong observational evidence to conclude that the active primary is the source of the radio emission in IM Peg. This result makes IM Peg only the second close binary system for which such an identification has unambiguously been made, the other being the close binary in the Algol system (Lestrade et al. 1993).

\subsection{Distribution of Position Residuals on the Sky}

We show in Figure 3(a) the residuals of our set (3) position solutions to our nine-parameter-weighted least-squares astrometric fit. The residuals correspond to the positions plotted in Figure 1 after the removal of the model orbit. In addition, we plot the disk of the primary, placing its center at the origin of the diagram. There are two important features in the sky distribution of the residuals: first, almost all of the residuals lie inside of the disk of the primary, with some residuals going only slightly beyond it. In fact, the 0.55 mas root-mean-square (rms) scatter ( 0.35 mas in $\alpha$ and 0.46 mas in $\delta$ ) is almost equal to the angular radius of the primary of $0.64 \pm 0.03$ mas (Table 1 ) but smaller than the semimajor axis of the orbit of the primary of $0.89 \pm 0.09$ mas (Table 2 ). With a relatively small mean standard error in the position determination of the stellar radio source of 0.07 mas in $\alpha$ and 0.09 mas in $\delta$, the scatter is largely intrinsic to the emission source. ${ }^{7}$ Second, the residuals are scattered preferentially along a northwest-southeast-oriented axis, approximately along the projected normal of the orbit. It is likely that this preferential elongation of the distribution of the residuals is also intrinsic to the emission source. The synthesized

\footnotetext{
6 This result depends on the assumption that there is no offset between the center of the primary and the mean position of the radio emission that is constant or steadily increasing/decreasing over the 8.5 years of VLBI observations. Such an offset could of course not be determined in our fit since it would be absorbed in our position and proper-motion estimates. However, as we demonstrate in Section 4.3, the distribution of our position solutions well covers the disk of the primary. Therefore, it appears that the emission locations are very closely linked to the primary and any constant or linearly changing offset of the center of this distribution from the center of the primary is likely smaller than the radius of the primary (see also Paper V).

7 The mean standard error in the position determinations is the root sum square of the mean statistical standard error associated with determining the position of the reference point in the image of IM Peg at each epoch and an estimated $\sim 0.06$ mas astrometric error in each IM Peg coordinate (Paper V). The astrometric error includes the rms "jitter" of 3C 454.3 component C1 relative to our extragalactic reference frame (see Paper III).
} 

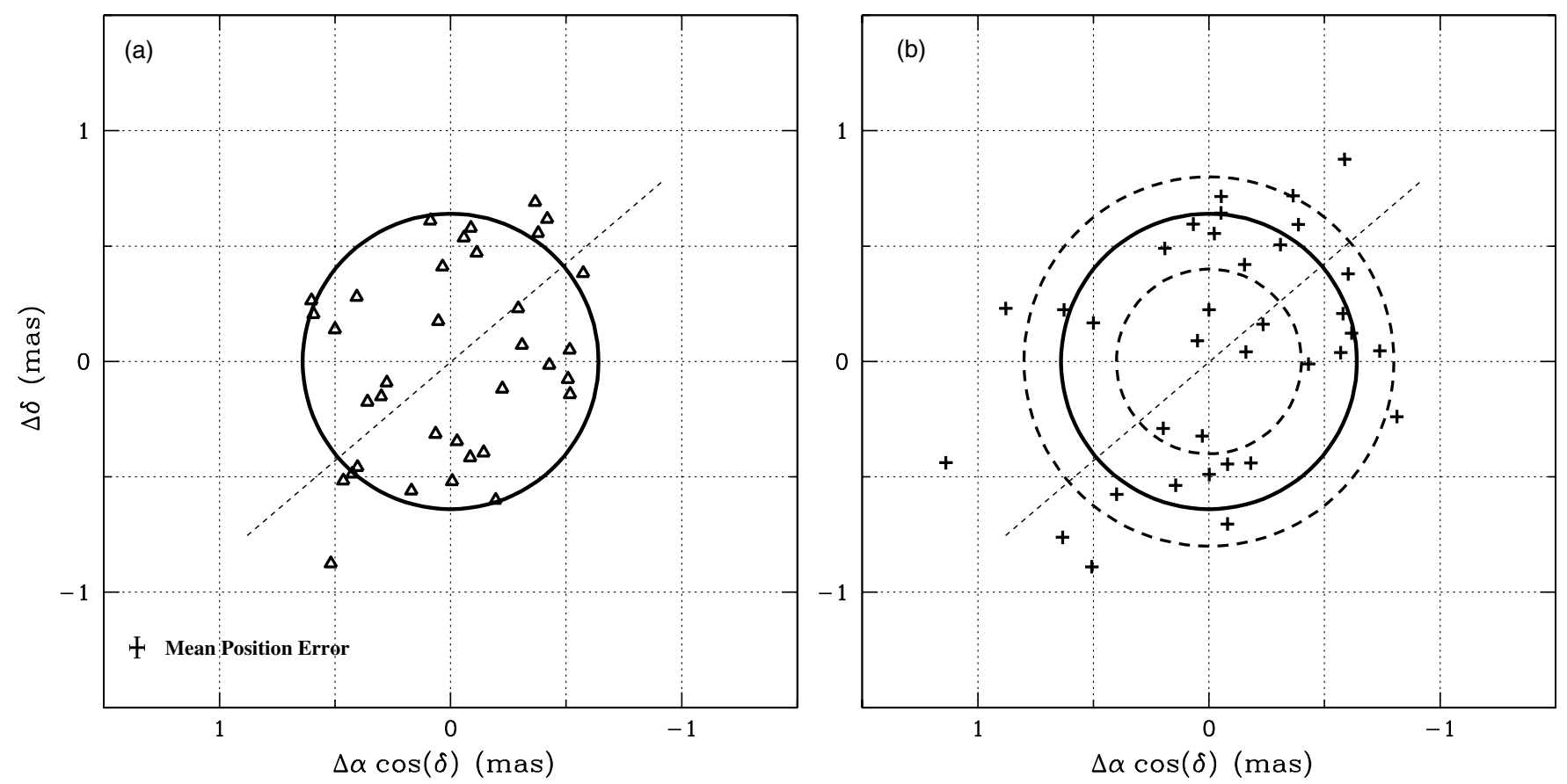

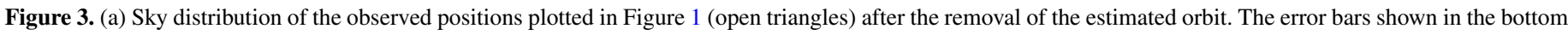

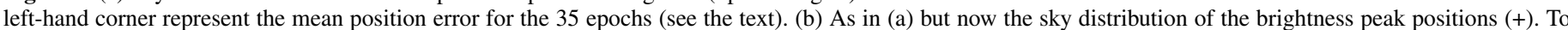

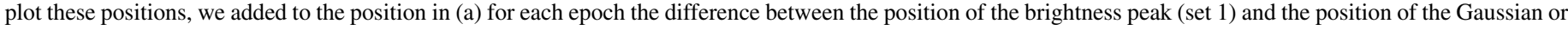

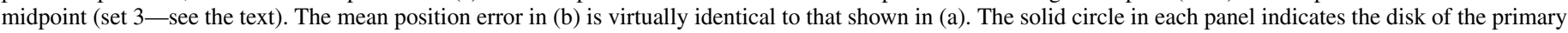

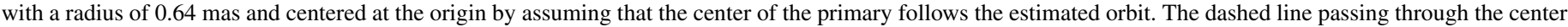

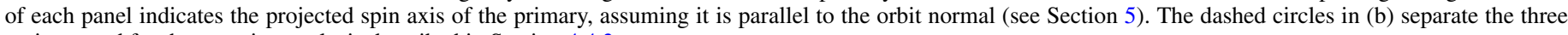
regions used for the counting analysis described in Section 4.4.3.

interferometer beam is by comparison more nearly oriented north-south (mean P.A. $\sim-7^{\circ}$ ). Moreover, when we fit for the purposes of error analysis the same nine-parameter model to the positions of the secondary reference source B2250+194 (which is $\sim 5$ times farther away from 3C 454.3 than IM Peg), we found an approximately threefold smaller scatter in the rms residuals and a smaller correlation between coordinates.

For the physical interpretation of the location of the source of radio emission, it is more meaningful to study the distribution of the positions of the brightness peaks (set 1) which indicate where the dominant part of the emission originates. We therefore added to the residual for each epoch the difference between the position of the interpolated brightness peak (from set 1) and the position of the Gaussian or midpoint (from set 3 ) used in our astrometric fit. Use of these "modified" residuals (hereafter referred to simply as residuals) ensures that we are plotting the offsets of the interpolated brightness peak at each epoch from our estimated orbit (see Figure 3(b)). The rms scatter about the mean is $0.62 \pm 0.03$ mas, and, as expected, somewhat larger than the rms scatter of 0.55 mas in Figure 3(a).

We determined the 0.03 mas standard error for the above sample estimate of rms scatter using a bootstrap method (see Efron \& Tibshirani 1993). More specifically, we regarded the 35 residuals as the parent distribution and chose from this distribution a new set of 35 residuals, with each one being randomly selected from the parent distribution (with replacement), until we obtained 500 different sets of 35 residuals. For our estimate of the standard error of the rms scatter of the true distribution, i.e., before sampling by our observations, we take the standard deviation of the 500 values of rms scatter that we computed from the 500 sets. We used this bootstrap method to derive standard errors for all parameters estimated from, or compared with, the 35 residuals, both here and, where relevant, hereafter.

\subsection{Simulation of the Distribution of Position Residuals on the Sky and Comparison with Observations}

How can the scatter of the locations of brightness peaks be interpreted in terms of the geometry of the orbit and the relation to the primary? In other words, how closely is the seemingly preferred direction of the scatter of these locations related to the normal of the orbit and the spin axis of the primary, and how far from the surface of the primary do the brightness peaks originate? Motivated by our astrometric solution and the distribution of the brightness peak locations in Figure 3(b), we constructed a three-dimensional model to simulate emission regions in the corona of the primary. We call this model the coronal emission model (CEM). We then considered different distributions of locations of emission regions to find the best match to our observations and thereby obtain a reasonable model for the location of the source of radio emission in IM Peg.

\subsubsection{Latitude-independent Coronal Emission Model with Spillover Emission}

We start with the latitude-independent version of the CEM. In this model, we assume that on average the radio emission is centered, in projection on the sky, on the center of the stellar disk, and allow the location of peak brightness to fall with equal probability above any point on the stellar surface. We allow emission to occur at any height above the photosphere of the star, but assume that the probability that the emission peaks at a given height decreases exponentially with scale height, $H^{8}$

\footnotetext{
8 We are not asserting here that an exponentially decreasing emission probability is physically realistic. An exponential function provides via a single parameter an analytical means of estimating the statistical distribution of emission heights. Other functions (e.g., uniform emission probability) employ a sharp cutoff at an arbitrary height and, more importantly, were not able to reproduce in projection the full extent and distribution (in the three regions described in Section 4.4.3) of the observed residuals.
} 
We then project the CEM onto the sky to compare our model distribution to the distribution of the locations of brightness peaks as plotted in Figure 3(b). For emission from locations not occulted by the disk of the star, we simply project that location onto the sky. For emission locations occulted by the disk of the star, we allow "spillover," i.e., we move the predicted location of observed peak emission outward along the radial line on the sky to a location we can see slightly beyond the edge of the stellar disk (see below). Allowing for spillover is perhaps reasonable, since we had a $100 \%$ detection rate and observed extended radio structure at every epoch; however, as we shall later show, nature does not seem to have followed this path and there are other ways to understand the $100 \%$ detection rate. We do not account in any sophisticated way for the transmission of spillover emission through the corona, e.g., by considering scattering, absorption, and/or refraction along the line of sight. Instead, we arbitrarily allow a small radial distribution of this emission on the sky spanning a projected radius of 1.0-1.1 times the stellar radius. The adjustable parameter in the latitude-independent CEM is $H$.

To execute the comparison, we computed several sets of 5000 locations each for the CEM, each set with the nominal value for the stellar radius but a different $H$. For each set, we compared the rms of the distribution of the radio emission locations to the $0.62 \pm 0.03$ mas rms scatter of the observed residuals. We found agreement between the two rms values for $H=0.11 \pm 0.03$ mas, i.e., a scale height only $0.17 \pm 0.05$ times the 0.64 mas stellar radius. The standard error in $H$ is the variation in that parameter needed to produce an increase or decrease in rms of the distribution of the radio emission locations equal to the standard error $( \pm 0.03$ mas $)$ of the rms of the scatter in the residuals. We provide an illustration of the latitude-independent CEM in Figure 4(a).

The figure illustrates that the latitude-independent CEM produces a circularly symmetric distribution of emission locations on the sky. Such a distribution fails qualitatively to describe the elongation in the scatter of the residuals. For a more direct comparison with the scatter of the 35 residuals, we produced several sets of 35 (random) emission locations from the latitudeindependent CEM, and for none of them was there more than a $16 \%$ difference between the rms along the line at which it was greatest from that along the line for which it was least. In contrast, the residuals show a $52 \% \pm 22 \%$ difference between the rms scatter along the line for which this rms scatter is maximum (p.a. $=-38^{\circ} \pm 8^{\circ}$ ) and the line for which this scatter is minimum.

\subsubsection{Latitude-dependent Coronal Emission Model with Spillover Emission}

To improve upon our CEM, we maintained our assumptions concerning spillover, but dropped our assumption of equal probability density per unit surface area. Instead, we allowed this probability density to vary with stellar latitude, $\lambda$. This enhancement of our model was motivated by Doppler optical surface images of the IM Peg primary which show persistent ( $\sim 1-3$ years and possibly longer), high-intensity spot features in both the mid-latitude range and directly over the visible pole (Berdyugina et al. 2000; Berdyugina \& Marsden 2006; Marsden et al. 2007). Given the close alignment expected for the spin axis and the orbit normal (see Section 5), we assumed that the spin axis of the primary is inclined $73^{\circ}$ to our line of sight, an angle equal to our VLBI-derived estimate of the inclination of the orbit (see Table 2). We tried a number of different functional forms for the latitude dependence of the probability density distribution. We found that a distribution proportional to $k+\sin |\lambda|$, with $k=0.14_{-0.04}^{+0.11}$, yielded a good fit to the sky distribution of the residuals. For this $k$ value, the mean probability density per unit surface area for emission near each pole $\left(|\lambda| \geqslant 70^{\circ}\right)$ is $3.6_{-0.7}^{+0.4}$ higher than that for emission near the equator $\left(|\lambda| \leqslant 20^{\circ}\right)$. The indicated $\sim 70 \%$ confidence limits reflect the uncertainty of the elongation of the scatter of the residuals, as estimated with our bootstrap approach.

We also varied the scale height, and found best agreement between the distribution of the CEM and the residuals for a value $0.15 \pm 0.05$ times the stellar radius. In Figure 4(b), we provide an illustration of the latitude-dependent CEM oriented on the sky with the spin axis lying along P.A. $=-38^{\circ}$, to align with the axis of the elongation of the distribution of our 35 residuals.

\subsubsection{Latitude-dependent Coronal Emission Model with Reduced Spillover Emission}

The latitude-dependent CEM with spillover emission gives us a distribution of projected emission points which is consistent with the distribution of residuals both in overall extent and degree of elongation. To compare the distributions in more detail, we looked at the number of emission points and residuals in each of three regions at increasing radial distances from the center of the distribution. Based on the extent of the distribution, we chose the following regions: (1) $<0.40$ mas, (2) $0.40-0.80$ mas, and (3) $>0.80$ mas. The regions are indicated by dashed circles in Figure 3(b) and in each of the panels in Figure 4. The results, given in Table 3, show that the number of emission locations in region 2 is significantly higher for the latitude-dependent CEM with spillover emission than for the observed residuals. Figure 4(b) shows clearly that, for the latitude-dependent CEM with spillover emission, many of the emission locations in region 2 are from spillover. Indeed, $35 \%$ of emission locations in this region and $28 \%$ of all points in this model are due to spillover. The percentage of instances for which the radio brightness peak arises from spillover emission may, however, be much lower. Here is why. In 9 of 35 epochs, our images of IM Peg show two (or three) local brightness maxima, with the maxima separated in 4 of those epochs by $\geqslant 0.5$ mas (see Paper VII). Since simultaneous emission from multiple regions appears common for IM Peg, it is reasonable to expect that spillover emission be dominated in many instances by emission from the Earthfacing side of the primary. To investigate this possibility, we modified the selection of projected emission locations in the latitude-dependent CEM with spillover emission. Instead of allowing each occulted location to become spillover emission, we allowed, first, only $50 \%$ of occulted locations to become spillover emission, replacing the other $50 \%$ with (randomly and independently drawn) nonocculted locations. We then decreased the fraction of allowed occulted locations to zero in intervals of $5 \%$ (i.e., $45 \%, 40 \%, .$. ), adding the deleted ones at nonocculted locations. For each such fraction, we adjusted the scale height, $H$, so as to maximize the agreement between the distribution of the CEM emission locations and that of the residuals. We found the best agreement between the distribution of emission locations in the CEM and that of the residuals, considering the number counts in the three regions defined above, for a reduced frequency of spillover emission with a fraction of $10 \% \pm 10 \%$ of allowed locations (see Table 3 ), and a scale height $0.20 \pm 0.05$ times the stellar radius. We provide in 

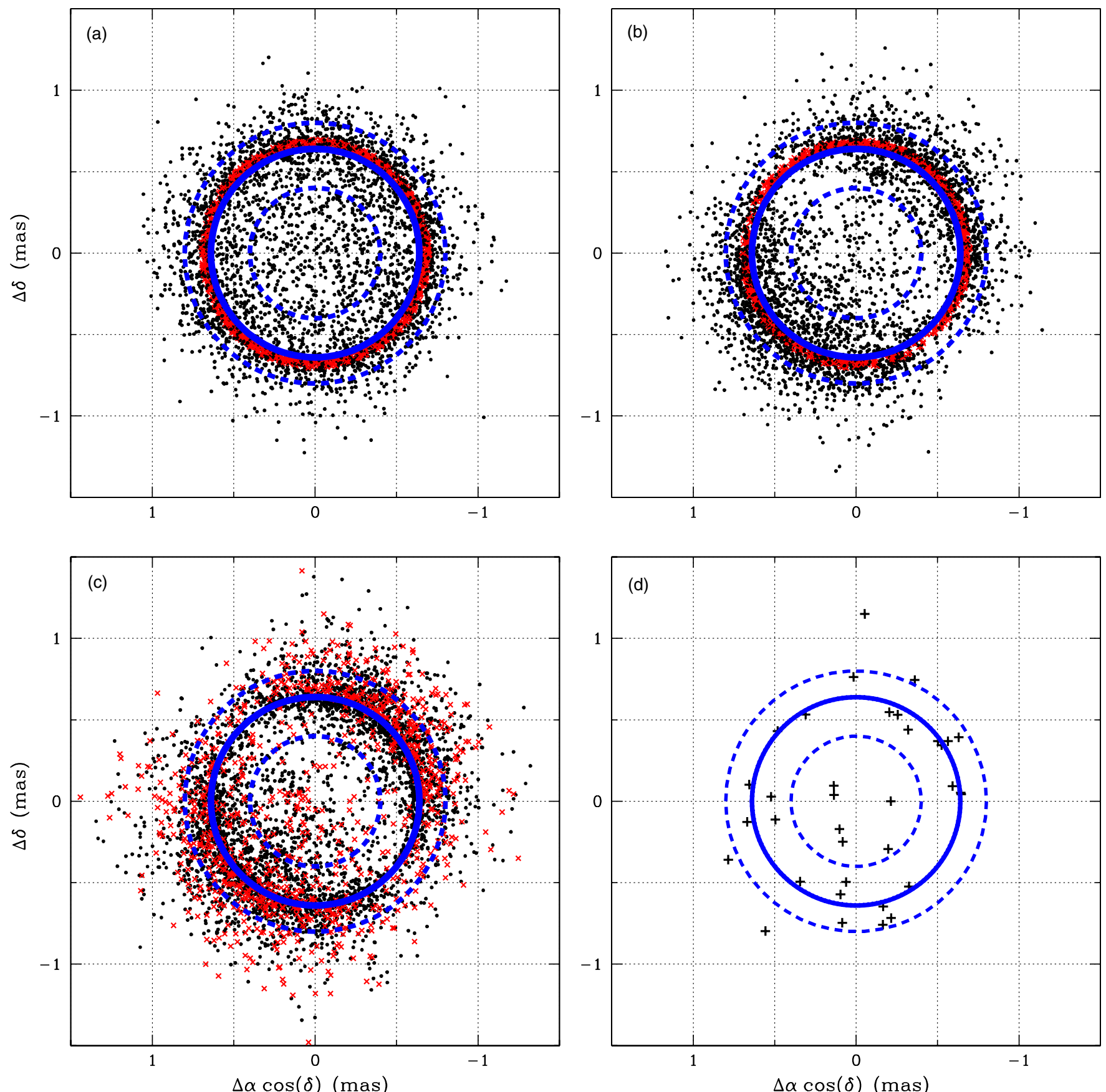

Figure 4. (a) Sky projection of 5000 random emission points from the latitude-independent CEM with spillover emission described in Section 4.4.1. The scale height in the model is 0.17 times the 0.64 mas stellar radius, a value which produced the best match to the observed distribution. The dots represent emission points that are not occulted by the disk of the star. The " $\times$ 's" (red in the colored version) just above the stellar disk represent spillover emission from points occulted by the disk of the star (see the text). (b) As in (a) but now the points from the latitude-dependent CEM with spillover emission described in Section 4.4.2. The scale height is 0.15 times the 0.64 mas stellar radius, again chosen so as to produce the best match to the observations. (c) As in (a) but now the points from the latitude-dependent CEM with reduced spillover emission described in Section 4.4.3. The scale height was 0.20 times the 0.64 mas stellar radius (again chosen for the best match). (d) Sky projection of 35 randomly selected emission points (+) from the latitude-dependent CEM with reduced spillover emission. For all panels, the solid circle gives the disk of the primary and the dashed circles separate the three regions used for the counting analysis described in Section 4.4.3.

(A color version of this figure is available in the online journal.)

Figure 4(c) an illustration of this latitude-dependent CEM, with reduced spillover emission, oriented on the sky with the spin axis lying along P.A. $=-38^{\circ}$. Note that for our best-fit model, in which the spillover from $10 \% \pm 10 \%$ of emission peaks in the occulted region yields an observed brightness peak, the percentage of all emission peaks which occur due to spillover is just $3 \% \pm 3 \%$. For a random sample of 35 emission locations, this value suggests that only $\leqslant 2$ of the locations arise from spillover emission. We show one realization of 35 randomly chosen emission points from the latitude-dependent CEM with reduced spillover emission in Figure 4(d).

\section{DISCUSSION}

Our astrometric result shows that the sources of radio emission are consistent with their being centered on average on the 
Table 3

Comparison of the Distribution of Residuals to the Distribution of CEM Emission Points

\begin{tabular}{lcrr}
\hline \hline & Residuals & Lat.dep. CEM with Spillover $^{\mathrm{a}}$ & Lat.-dep. CEM with Reduced Spillover $^{\mathrm{b}}$ \\
Region & 35 Epochs & 35 Points $^{\mathrm{c}}$ & 35 Points $^{\mathrm{c}}$ \\
\hline Region 1 & $6 \pm 2(17 \% \pm 6 \%)$ & $2 \pm 1(6 \% \pm 3 \%)$ & $5 \pm 2(14 \% \pm 6 \%)$ \\
Region 2 & $22 \pm 3(63 \% \pm 9 \%)$ & $29 \pm 2(83 \% \pm 6 \%)$ & $24 \pm 3(69 \% \pm 9 \%)$ \\
Region 3 & $7 \pm 3(20 \% \pm 9 \%)$ & $4 \pm 2(11 \% \pm 6 \%)$ & $7 \pm 2(20 \% \pm 6 \%)$ \\
\hline
\end{tabular}

Notes.

${ }^{a}$ Latitude-dependent CEM with spillover emission as discussed in Section 4.4.2.

b Latitude-dependent CEM with reduced spillover emission as discussed in Section 4.4.3. The tabulated results correspond to a 10\% allowed fraction of spillover points.

c The value and standard error given for each region represent the mean number and standard deviation for 500 realizations of 35 randomly chosen CEM emission points (see the text).

primary, and also consistent with theoretical models which propose that the radio emission in active close binaries is powered and confined by the magnetic field of the active star (see, e.g., Lestrade et al. 1988; Mutel et al. 1998; Franciosini et al. 1999). Any constant or linearly changing offset of the center of the distribution of emission locations from the center of the disk of the primary over the 8.5 years of our VLBI observations is not necessarily expected to be zero. First, any latitude-dependent emission model when combined with shadowing of one of the pole regions due to the inclination of the spin axis would predict a constant offset. Second, a systematic time dependence of the latitude distribution of spot centers could arise given the apparent multi-year stellar-activity cycle of the IM Peg primary (e.g., Berdyugina et al. 2000; Zellem et al. 2010). With the distribution of the radio emission locations at least partly linked (see below) to such spot features, an offset with a nonzero trend from 1997 to 2005 could plausibly contribute error to our proper-motion estimate. However, each of these possible causes would not result in the offset being larger than the radius of the primary (see Paper V). This estimate, together with the distribution's matching the disk of the primary, makes us believe that any offset at any time during our observations is indeed smaller than the radius of the primary.

Our position residuals are scattered preferentially along an axis with P.A. of $-38^{\circ} \pm 8^{\circ}$, which, to within the combined uncertainties, is equal to the P.A. of the sky-projected orbit normal of $-49.5 \pm 8.6$. Since the orbit normal is expected to be closely aligned with the spin axis of the primary as for all synchronous RS CVn systems (see Stawikowski 1994), the sources of radio emission appear to be linked to the spin axis of the primary. Indeed, a comparison of the scatter of the position residuals with our simulations shows that the probability density per unit surface area for radio brightness peaks is $3.6_{-0.7}^{+0.4}$ higher near the poles $\left(|\lambda| \geqslant 70^{\circ}\right)$ than near the equator $\left(|\lambda| \leqslant 20^{\circ}\right)$. Since Doppler images of IM Peg show the presence of persistent, high-intensity spot features at the pole region of the primary (Berdyugina \& Marsden 2006; Marsden et al. 2007), our result provides statistical evidence that the radio emission regions occur at the same stellar latitudes as do active surface regions.

Our simulations place restrictions on the altitude of the locations of radio-emitting structures in the corona of the IM Peg primary. In our best-fit model, $\sim 2 / 3$ of the brightness peaks arise within an altitude of just 0.25 times the stellar radius. Since the stellar magnetic field is presumably strongest near the surface, this result may not be surprising. However, it is different from the pictures presented for the close binary systems Algol (e.g., Lestrade et al. 1988) and UX Arietis (e.g., Franciosini et al.
1999), which show emission from magnetic-loop structures with heights greater than one stellar radius. If emission does occur high on magnetic loops for IM Peg, then most of these loops are small in height compared to the radius of the primary.

Our simulations further allowed us to set at $6 \%$ the $(1 \sigma)$ limit on the likelihood (at any given epoch) that the brightness peak arises from spillover. Although this limit is model-dependent, we think that the more general conclusion, which is that the observed brightness peaks arise mostly from emission regions connected to the Earth-facing side of the primary, is robust. Spillover could, however, be responsible for lowersurface-brightness features of the radio structure. In many of our epochs, the IM Peg radio structure is multi-peaked or at least significantly elongated. While the highest peak in these instances is likely to be associated with emission from the Earthfacing side, spillover could very well contribute to the overall shape. In Paper VII, we present the full set of IM Peg images with the outlines of the disk and orbit of the primary superimposed, and discuss in detail the size and shape of the radio emission regions.

\section{CONCLUSIONS}

Here, we summarize our results and give our conclusions.

1. The sources of radio emission are on average located near the center of the disk of the primary, in particular being within $12 \% \pm 15 \%$ of its radius of $0.64 \pm 0.03$ mas, provided any 8.5 year constant or linearly changing offset is (nearly) zero. There are theoretical arguments as well as observational evidence that any such offset is indeed smaller than the radius of the primary. Thus, IM Peg is the second close binary with such (nearly) unambiguous identification.

2. The positions of the sources of observed radio emission are scattered over an area on the sky slightly larger than the disk of the primary and preferentially along an axis with P.A. $=-38^{\circ} \pm 8^{\circ}$. This axis is closely aligned with the skyprojected orbit normal (P.A. $=-49.5 \pm 8.6$ ) and expected spin axis of the primary.

3. Comparison of our observed positions with simulations suggests that the radio brightness peaks are more likely to occur at higher stellar latitudes than near the stellar equator, with the probability density per unit surface area being $3.6_{-0.7}^{+0.4}$ times higher near the poles $\left(|\lambda| \geqslant 70^{\circ}\right)$ than near the equator $\left(|\lambda| \leqslant 20^{\circ}\right)$. The radio emission regions therefore show a dependence on stellar latitude similar to that exhibited by active regions on the primary's surface seen as dark spots with optical Doppler imaging. 
4. This comparison also suggests that these brightness peaks preferentially arise close to the surface of the primary, with $\sim 2 / 3$ of them located no more than 0.25 stellar radii above the surface.

5. This comparison further suggests that the brightness peaks are mostly associated with emission regions on the Earthfacing side of the primary, with peaks caused by spillover from emission regions on the opposite side of the star occurring rarely, if ever.

We thank the anonymous referee for a constructive review of the paper and for comments helpful in the preparation of the final manuscript. This research was primarily supported by NASA, through a contract with Stanford University to SAO, and a subcontract from SAO to York University. The VLBA is an instrument of the National Radio Astronomy Observatory (NRAO), a facility of the National Science Foundation operated under cooperative agreement by Associated Universities, Inc. The DSN is operated by JPL/Caltech, under contract with NASA. We have made use of NASA's Astrophysics Data System Abstract Service, developed and maintained at SAO. Jeff Cadieux and Julie Tomé assisted with the reduction of the VLBI data during their tenures as students at York University.

\section{REFERENCES}

Bartel, N., Bietenholz, M. F., Lebach, D. E., et al. 2012, ApJS, 201, 3 (Paper III) Bartel, N., Herring, T. A., Ratner, M. I., Shapiro, I. I., \& Corey, B. E. 1986, Nature, 319, 733

Beasley, A. J., \& Conway, J. E. 1995, in ASP Conf. Ser. 82, Very Long Baseline Interferometry and the VLBA, ed. J. A. Zensus, P. J. Diamond, \& P. J. Napier (San Francisco, CA: ASP), 327

Berdyugina, S. V., Berdyugin, A. V., Ilyin, I., \& Tuominen, I. 2000, A\&A, 360, 272

Berdyugina, S. V., Ilyin, I., \& Tuominen, I. 1999, A\&A, 347, 932

Berdyugina, S. V., \& Marsden, S. C. 2006, in ASP Conf. Ser. 358, ed. R. Casini \& B. W. Lites (San Francisco, CA: ASP), 385
Bietenholz, M. F., Bartel, N., Lebach, D. E., et al. 2012, ApJS, 201, 7 (Paper VII) Boboltz, D. A., Fey, A. L., Johnston, K. J., et al. 2003, AJ, 126, 484 Dempsey, R. C., Neff, J. E., O’Neal, D., \& Olah, K. 1996, AJ, 111, 1356 Drake, S. A., Simon, T., \& Linsky, J. L. 1989, ApJS, 71, 905

Efron, B., \& Tibshirani, R. J. 1993, An Introduction to the Bootstrap (Boca Raton, FL: Chapman and Hall/CRC)

ESA 1997, The Hipparcos and Tycho Catalogues (ESA SP-1200; Noordwijk, Netherlands: ESA)

Franciosini, E., Massi, M., Paredes, J. M., \& Estalella, R. 1999, A\&A, 341, 595 Hall, D. S. 1976, in IAU Colloq. 29, Multiple Periodic Variable Stars, ed. W. S. Fitch (ASSL, Vol. 60; Dordrecht: Reidel), 287

Huenemoerder, D. P., Ramsey, L. W., \& Buzasi, D. L. 1990, ApJ, 350, 763

Lebach, D. E., Bartel, N., Bietenholz, M. F., et al. 2012, ApJS, 201, 4 (Paper IV)

Lebach, D. E., Ratner, M. I., Shapiro, I. I., et al. 1999, ApJ, 517, L43

Lestrade, J.-F. 1996, in IAU Symp. 176, Stellar Surface Structure, ed. K. G. Strassmeier \& J. L. Linsky (Cambridge: Cambridge Univ. Press), 173

Lestrade, J.-F., Jones, D. L., Preston, R. A., et al. 1995, A\&A, 304, 182

Lestrade, J.-F., Mutel, R. L., Preston, R. A., \& Phillips, R. B. 1988, ApJ, 328, 232

Lestrade, J.-F., Phillips, R. B., Hodges, M. W., \& Preston, R. A. 1993, ApJ, 410, 808

Lestrade, J.-F., Preston, R. A., Jones, D. L., et al. 1999, A\&A, 344, 1014

Lestrade, J.-F., Rogers, A. E. E., Whitney, A. R., et al. 1990, AJ, 99, 1663

Marsden, S. C., Berdyugina, S. V., Donati, J.-F., Eaton, J. A., \& Williamson, M. H. 2007, Astron. Nachr., 328, 1047

Marsden, S. C., Berdyugina, S. V., Donati, J.-F., et al. 2005, ApJ, 634, L173

Morris, D. H., \& Mutel, R. L. 1988, AJ, 95, 204

Mutel, R. L., Lestrade, J. F., Preston, R. A., \& Phillips, R. B. 1985, ApJ, 289, 262

Mutel, R. L., Molnar, L. A., Waltman, E. B., \& Ghigo, F. D. 1998, ApJ, 507, 371

Olah, K., Marik, D., Houdebine, E. R., Dempsey, R. C., \& Budding, E. 1998, A\&A, 330, 559

Ransom, R. R., Bartel, N., Bietenholz, M. F., et al. 2002, ApJ, 572, 487

Ransom, R. R., Bartel, N., Bietenholz, M. F., et al. 2012, ApJS, 201, 2 (Paper II)

Ratner, M. I., Bartel, N., Bietenholz, M. F., et al. 2012, ApJS, 201, 5 (Paper V)

Shapiro, I. I., Bartel, N., Bietenholz, M. F., et al. 2012, ApJS, 201, 1 (Paper I)

Shapiro, I. I., Wittels, J. J., Counselman, C. C., III, et al. 1979, AJ, 84, 1459

Spangler, S. R., Owen, F. N., \& Hulse, R. A. 1977, AJ, 82, 989

Stawikowski, A. 1994, Acta Astron., 44, 393

Uchida, Y., \& Sakurai, T. 1983, in IAU Colloq. 71, Activity in Red-Dwarf Stars, ed. P. B. Byrne \& M. Rodono (ASSL, Vol. 102; Dordrecht: Reidel), 629

Zahn, J.-P. 1977, A\&A, 57, 383

Zellem, R., Guinan, E. F., Messina, S., et al. 2010, PASP, 122, 670 